REVISTA DEESTUDIOS BRASILEÑOS

e-ISSN: $2386-4540$

https://doi.org/10.14201/

reb2019611155167

AUTOR

César Augusto

De las Casas*

cdelascasas58@

gmail.com

* Ministro en el Servicio Diplomático del Perú. MSc en PWE por la

LSE (RU), MSocSc por la Universidad de Estocolmo (Suecia).

Exdirector Ejecutivo de la SP/OTCA.

\section{El bioma amazónico y el Acuerdo de París: cooperación y gobernanza}

\author{
O bioma amazônico e o Acordo de Paris: cooperação e governança
}

The Amazon biome and the Paris Agreement: Cooperation and Governance

\section{RESUMEN:}

Se plantea una nueva conceptualización en el modo de abordar la relación entre bioma amazónico y cambio climático, particularmente respecto a la implementación del Acuerdo de París sobre Cambio Climático y a la construcción de una buena gobernanza climática, para lo cual se propone la formulación de los sistemas social-ecológicos Tierra y Amazonia, respectivamente, como dos unidades distintas, pero conectadas para el análisis y la gestión de los problemas descritos, y cuya relación es fundamental para la construcción de la resiliencia. En ese contexto se destaca el papel de los pueblos indígenas de la Amazonia como protagonistas históricos de la adaptación y transformación del paisaje amazónico, dando prioridad a la necesidad de reconocer que las políticas públicas deben ser coherentes al integrar las políticas de mitigación y de adaptación en un sentido transformador.

\section{RESUMO:}

Propõe-se a necessidade de uma nova conceituação no sentido de abordar a relação entre o bioma amazônico e as mudanças climáticas, particularmente considerando a implementação do Acordo de Paris sobre Mudança do Clima e a construção de uma boa governança climática. Os sistemas social-ecológicos Terra y Amazônia, respectivamente, são propostos como duas unidades distintas, mas conectadas para a análise e gestão dos problemas descritos, e cuja relação é fundamental para a construção da resiliência. Nesse contexto, destaca-se o papel dos povos indígenas da Amazônia como protagonistas históricos da adaptação e transformação da paisagem amazônica, revelando a necessidade de reconhecer que as políticas públicas devem ser coerentes ao integrar as políticas de mitigação e adaptação em um sentido transformador.

\footnotetext{
ABSTRACT:

A new conceptualization is proposed for approaching the relationship between the Amazon biome and climate change, particularly with respect to the implementation of the Paris Agreement on Climate Change and the construction of a good climate governance. In that regard, the Earth and the Amazon are socialecological systems to be considered as two different but connected units of analysis and management of the problems described, and fundamental for the construction of resilience. In this context, the role of indigenous peoples of the Amazon as protagonists of the adaptation and transformation of the Amazonian landscape is highlighted, as well as the need to recognize that public policies must be coherent when integrating mitigation and adaptation policies in a transformative sense.
} 


\section{Introducción}

La existencia de diversos acuerdos internacionales en materia ambiental ha sido descrita como una situación de fragmentación que no permite la construcción de una gobernanza ambiental a nivel global. Esa postura es contestada por otros, en el sentido que la naturaleza compleja y variada de los biomas no permitiría la existencia de una gobernanza ambiental única.

El tema, no obstante, no se agota simplemente en la integración de los distintos regímenes ambientales internacionales, sino que se requiere particularmente integrar dentro de ella a los regímenes internacionales económicos. En otras palabras, los modos de producción y consumo no-sostenibles y las formas en las que la economía, finanzas, comercio y actividades energéticas globales funcionan, son factores estructurales. Estos factores deben ser reformados dentro de esa amplia gobernanza climática global, si es que quiere avanzar. De lo contrario, poco o nada se podrá conseguir respecto a la reducción de las emisiones de gases de efecto invernadero (GEI), pese al esfuerzo que se pueda realizar mediante acciones de mitigación, adaptación y construcción de resiliencia.

El mismo problema, pero a una escala regional, surge cuando se intenta abordar al bioma amazónico y a la Amazonía y sus problemas en general. Esa fragmentación se expresa, entonces, en enfoques de análisis y de gestión sectoriales, sin conseguir una visión coherente que tome en cuenta que la Amazonía es una unidad o sistema social-ecológico complejo.

Dentro de ese contexto, se quiere abordar la relación entre el bioma amazónico y el Acuerdo de París sobre Cambio Climático (APCC), con respecto a las tareas que se tendría por delante para su implementación, desde una perspectiva general y regional. Si no se procede de esta forma, sólo obtendremos una visión segmentada, parcial e incompleta de la Amazonía, sin considerar sus dimensiones geográficas, económicas, sociales, culturales, políticas o social-ecológicas.

El bioma amazónico es un componente principal dentro del sistema social-ecológico que es la Amazonía, entendida como la unidad de análisis y de gestión. Ese sistema social-ecológico integra, además, la dimensión espacial, que recoge las escalas geográficas del análisis y gestión: amazónico-local, amazónico-regional y amazónico-global. Metodológicamente, hay una relación entre las escalas global, regional y local que debe integrarse en el análisis, especialmente si queremos abordar la relación entre un bioma concreto como el amazónico y un instrumento jurídico global, como es el APCC, cuyo propósito como todo Tratado, no es regular el ecosistema en sí mismo, sino la conducta humana respecto al cambio climático (Young, 2007), lo cual no es una tarea simple.

Se integra de este modo la acción humana con la naturaleza y la infinidad de sinergias que pueden generarse entre sí, como es la construcción de resiliencia social-ecológica frente al cambio climático.

Asimismo, la Amazonía como unidad de análisis y de gestión se justifica por sus dimensiones espaciales y funciones/servicios ecosistémicos. Su papel global en el ciclo del carbón es importante como sumidero de carbono. Lo es también en el hidrológico a través del proceso de evapotranspiración ${ }^{1}$ del bosque, y el desplazamiento de masas de vapor de agua, denominados ríos voladores o atmosféricos, hacia el interior de la Amazonía u de otras regiones fuera de ella. La existencia del bosque húmedo tropical, la cuenca hidrográfica del río Amazonas - principal fuente de agua dulce del mundo - la mega- diversidad biológica, y la diversidad cultural y social, entre otros aspectos, son características relevantes. Adicionalmente, se justifica por su importancia respecto a los otros ecosistemas regionales y globales. 
En conclusión, por sus dimensiones la Amazonía es un sistema social-ecológico complejo, esto es, caracterizado por ser no-lineal y de escalas múltiples. Por ello, conceptualizar a la Amazonía como unidad de análisis y de gestión, implica utilizar una perspectiva holística e integrada que sepa reconocer a la Amazonía como sistema social-ecológico² (Berkes \& Folke, 2003), en donde la naturaleza y la persona humana son partes dentro de una unidad en permanente transformación.

Adicionalmente, dentro de esa conceptualización social-ecológica de la Amazonía, se valoriza el papel de los conocimientos tradicionales ${ }^{3}$ de los pueblos indígenas y comunidades amazónicas locales. En esta perspectiva, se reconoce, además, que las mujeres y hombres de la Amazonía son sujetos de derechos, que aspiran a una mejor calidad de vida, en un contexto caracterizado por problemas de orden social y económico, como la pobreza, desigualdad, deforestación, las actividades ilegales y la degradación ambiental, entre otras lacras que afectan a la Amazonía. Estas y otras condiciones contextualizan la situación en la que el bioma amazónico se enfrenta a los efectos adversos del cambio climático.

\section{El bioma amazónico}

El bioma amazónico posee un territorio variado que se caracteriza por la diversidad de sus entornos naturales, biológicos, geográficos, sociales y culturales. Está dividido "en la selva baja o llano amazónico, la selva alta, y la ceja de selva o yungas, drenada por el río Amazonas que es el más largo del mundo y con la cuenca hidrográfica más extensa, con más de 1.000 tributarios, la Amazonía alberga una gran variedad de especies de flora y fauna, y es un área importante de endemismos. Por otro lado, la Amazonía es también sinónimo de diversidad cultural, con 420 pueblos indígenas distintos, 86 lenguas y 650 dialectos" (PNUMA et al., 2009).

El bioma amazónico es el bosque tropical más extenso del planeta, con alrededor de siete millones de kilómetros cuadrados, que incluye parte importante del territorio de ocho países. Su importancia como un gran ecosistema es de alcance planetario, siendo que el río Amazonas es el más largo, aportando aproximadamente el veinte por ciento del agua dulce del mundo al Océano Atlántico. Es un ecosistema regulador del dióxido de carbono $\left(\mathrm{CO}_{2}\right)$ y productor de oxígeno a nivel planetario, sin llegar a ser el "pulmón del mundo"; función que, en realidad, corresponde a un arrecife de corales ubicado en Australia ${ }^{4}$.

El bioma Amazónico tiene un papel en el equilibrio climático global, pues permite una estupenda regulación de las lluvias al mantener y posibilitar continuamente el ciclo del agua, fenómeno que hace de la Amazonía un ambiente caluroso y muy húmedo. Esto último se debe a que constantemente la acción del sol, con su temperatura superior a los $25^{\circ} \mathrm{C}$, provoca la evaporación del agua de las lluvias para formar nubes que contribuirán con nuevas precipitaciones, inclusive en otros lugares del planeta (PNUMA et al., 2009).

Según el Informe del Desarrollo 2010 del Banco Mundial (World Bank, 2010): "Los ecosistemas más críticos de América Latina y el Caribe están bajo amenaza", ya que "el impacto más desastroso podría ser una dramática muerte regresiva de la selva amazónica y una conversión de grandes áreas a la sabana, con graves consecuencias para el clima de la región, y posiblemente del mundo".

Observando el conjunto de la Región Amazónica, encontramos que el crecimiento de los centros urbanos ha llevado a la población a alcanzar aproximadamente los 40 millones de habitantes. Hay un crecimiento superior a la tasa promedio de los ocho países, siendo que en los 1970s era de sólo 5 millones, lo que tiene que ver también con las migraciones internas y con las políticas gubernamentales de colonización que fueron promovidas (PNUMA \& OTCA, 2009).

Asimismo, el bioma amazónico sufre la presión de las actividades productivas, especialmente aquellas destinadas a atender la demanda internacional de commodities como los hidrocarburos, la madera y la 
minería por extracción ${ }^{5}$ no-sostenible. Adicionalmente, tenemos que las carreteras y las grandes obras de infraestructura y el cambio del uso de los suelos, aceleran la pérdida de las funciones y servicios ecosistémicos, y contribuyen a la fragmentación de los ecosistemas, la deforestación y la pérdida de la biodiversidad (PNUMA \& OTCA, 2009).

Según el IPCC (2014), el cambio climático tiene, en general, un mayor "impacto en los sistemas naturales". En el caso de la Amazonía, el "impacto en los sistemas humanos" es particularmente importante debido a que la población local depende más de su entorno natural y es más vulnerable a cualquier cambio o variabilidad climática. La recolección de alimentos, la caza, la agricultura de subsistencia y la pesca son actividades esenciales para la nutrición y la supervivencia de los pueblos indígenas. Los pueblos indígenas en la Amazonia "dependen mucho del bosque" y han tenido que implementar medidas de adaptación como diversificación de cultivos, migración a áreas más seguras, cambios en las rutinas de caza que se ajustan a los nuevos desplazamientos de animales y muchos más (UICN, 2008, p. 826). Finalmente, incluso si tratamos de recuperar tierras degradadas mediante reforestación, "el riesgo de una pérdida significativa de biodiversidad por la extinción de especies en muchas áreas" no es reversible en absoluto, con grandes efectos catastróficos en otros bosques vecinos, agricultura y seguridad alimentaria (Tanner et al., 2014, p. 39). Como lo describe Fearnside (2009, p.1004) el "cambio climático es una amenaza al bosque amazónico y la pérdida del bosque es una amenaza al clima".

Por ello, al adoptar un enfoque basado en la Amazonía como sistema social-ecológico y como unidad de análisis y de gestión, estamos asumiendo que la acción de las mujeres y hombres de la Amazonía ha tenido, a lo largo de la historia, funciones tanto de adaptación a su entorno cuanto de transformación del mismo. Esas funciones de adaptación y trasformación se dan a través del devenir histórico que, se sintetiza, en cómo la Amazonía como sistema social-ecológico se articuló subordinadamente a la economía capitalista internacional desde el Siglo XVI, lo que revela las condiciones en las que el fenómeno del cambio climático se desarrolla y que subyacen como causas económicas y materiales del mismo. En ese sentido, no basta el enfoque sostenido por Crutzen (2002) sobre el antropoceno, que limita el surgimiento de éste a la Revolución Industrial y al uso masivo de combustibles fósiles desde el Siglo XIX, sino que es necesaria una aproximación de más largo plazo que incluye la ocupación y apropiación del espacio.

Como señala Gonçalves (2012, pp. 1397-1403), la ocupación de la Amazonía se da en el marco de las disputas geopolíticas coloniales entre españoles, portugueses, holandeses, ingleses y franceses, siendo que el interés por las riquezas de la Amazonía sólo irá creciendo en el tiempo para los europeos, que la veían inicialmente como una reserva de recursos en comparación con otros territorios coloniales más atractivos y accesibles para su explotación comercial inmediata.

En ese sentido, la ocupación y apropiación europea se da atendiendo a los intereses de los grandes actores en el mercado mundial, articulando económicamente en esa dinámica al espacio amazónico. Posteriormente, la geopolítica asumirá el papel de enfoque principal frente a un territorio inicialmente desconocido y extenso, estableciendo mecanismos de control territorial soberano sobre puntos estratégicos, al servicio de intereses políticos y económicos concretos.

El principio del uti possidetis y los derechos soberanos de las potencias coloniales, devienen en las herramientas jurídicas principales que justifican la progresiva posesión de los territorios. Esta conceptualización exógena que privilegia la dominación externa, se desarrolla paralelamente a otra basada en una lógica interna o endógena que privilegiaba el desarrollo y las necesidades de los pueblos indígenas y poblaciones locales, como es el ejemplo de las misiones jesuitas y carmelitas (Becker, 2013, p. 136).

Gonçalves (2012, pp. 1376-1380) describe dos formas en las que el espacio amazónico fue organizado, y cuya influencia puede ser vista hoy en los paisajes actuales. Por un lado, desde la primera ocupación humana hasta la década de los 1960s, el espacio estaba organizado "del espacio río - tierras bajas o 
inundables - bosques". Por otra parte, desde la década de 1960s, el espacio amazónico se organiza como "espacio carretero - tierra firme - subsuelo". La explotación del subsuelo se da en los proyectos de explotación minera y un "nuevo flujo de materia y de energía en la región" bajo la tutela del estado y de los grandes capitales. Se produce una transformación socio-espacial que trae consigo el conflicto entre distintas valoraciones de los recursos (Gonçalves, 2012, pp. 1390-1393). Este proceso se va acelerar con el boom del caucho, conectando a la Amazonía con la Revolución Industrial y sus formas de explotación y opresión de la fuerza de trabajo indígena (Gonçalves, 2012, pp. 1543-1547).

Hay, en ese sentido, una ocupación y organización del espacio "que se ha plasmado por medio de un intercambio orgánico con los ecosistemas: el río, vertedero natural de toda el agua que circula a través del bosque, suelo y atmósfera, que sirvió para diferentes actividades que se desarrollaron explorando el bosque, los campos y las tierras bajas ${ }^{6}$. Fue por los ríos que se garantizó la conquista de la región. Esa Amazonía será apropiada bajo la nueva dinámica del capitalismo globalizado, imponiendo el modelo de organización "espacio carretero - tierra firme - subsuelo" (Gonçalves, 2012, pp. 1742-1745).

\section{La gobernanza climática}

Conforme a las corrientes académicas predominantes, la gobernanza puede emerger como una necesidad impuesta por cambios en la realidad. Esto ocurre, especialmente, cuando las actividades de ciertos individuos o colectividades crecen y desbordan las capacidades institucionales existentes. Por eso, en "las grandes sociedades modernas, la gobernanza es una preocupación crítica de la sociedad". En ese sentido, hay que tener en cuenta que la existencia de un gobierno formalmente constituido, no significa que el mismo esté en condiciones de cumplir, de modo efectivo, las funciones de esa gobernanza en una serie de situaciones. De hecho, la complejidad de los problemas ha requerido que algunos actores no-estatales hayan asumido en varias situaciones el liderazgo de dicha gobernanza (Young, 2017, pp. 4-5).

La gobernanza global es entendida también como una crítica a la ineficiencia y parálisis de la diplomacia multilateral y organismos internacionales, así como un llamado a su reforma (Biermann, 2014, p. 27). El concepto de gobernanza global implica la convergencia de individuos y colectividades de diverso origen, que son parte de una red de trabajo, y están interesados en la solución de un problema que, por su complejidad, no puede ser resuelto únicamente por el estado. Es el caso del cambio climático que emerge como una amenaza a la seguridad humana (Jang et al., 2016, p. 2).

La gobernanza del sistema Tierra pretende ser no sólo una gobernanza ambiental, sino incluir a "los procesos y prácticas sociales", dentro de un propósito normativo. Esta gobernanza al proponerse metas ambientales, buscaría su implementación a través de "varios mecanismos de gobernanza global y local, con costos diferenciados para los actores en distintos países y regiones” (Biermann, 2014, p. 28).

Biermann et al. (2010, p. 3) identifica tres temas centrales, no excluyentes entre sí, a ser desarrollados en relación a la gobernanza global del cambio climático: 1) la arquitectura de la gobernanza global del cambio climático, que promueve nuevas formas institucionales con una dirección no-estatal o colegiada entre estados y no-estados, que no está basada en tratados, y que utiliza esquemas más flexibles; 2) el surgimiento de la agencia o de nuevos actores no-estatales en la gobernanza del cambio climático; y, 3) la adaptación de las instituciones y organizaciones internacionales al cambio climático, que se expresa también en la fragmentación de la gobernanza global en diversos temas y funciones. Dicha diversidad temática refleja la complejidad de los temas y de los actores en juego en el cambio climático (Jang et al., 2016, p. 3).

Aunque la gobernanza global se puede mostrar a veces débil e inefectiva frente a los problemas, "la tendencia hacia la globalización y la demanda por enfoques basados en la gobernanza global ha pasado 
ya el punto de no retorno. El futuro de la gobernanza global estará conformado por los siguientes cinco factores: empoderamiento individual; creciente toma de conciencia de la seguridad humana; complejidad institucional; cambios de orientación en el poder internacional, y un paradigma político liberal mundial" (Jang et al., 2016, p. 3).

El calentamiento global coloca al planeta y sus habitantes ante el desafío de asegurar la sobrevivencia de la especie humana y su hábitat. En ese sentido, la gobernanza global del cambio climático (CMNUCC) en el que la cuestión de la implementación de medidas de mitigación y adaptación sea planteada, es estratégica, abriendo un espacio para la convergencia de esfuerzos de diversos actores estatales y no-estatales. Esta situación es particularmente notoria en el caso de las negociaciones de los acuerdos y convenciones ambientales y, más recientemente, en el Acuerdo de París sobre Cambio Climático (APCC). Se trasciende a las negociaciones propiamente dichas, para situarse dentro del proceso de toma de decisiones gubernamentales, en el diseño de políticas públicas y/o la implementación de proyectos de desarrollo sostenible. En general, el tema de la gobernanza climática emerge cada vez que el modelo de gobierno centralizado y de estado-centralista, no es considerado suficiente para responder a los desafíos y complejidades de los ecosistemas.

Pero la pregunta es, entonces: ¿cuál es el tipo de gobernanza más conveniente? ¿Puede ser uno en el que la expansión de una red ecológica/climática asegure que los diálogos políticos, sociales y económicos interactúen en la formulación de las políticas de conservación y desarrollo sostenible de los estados?

Al respecto, es importante que dicha gobernanza pueda garantizar la coherencia y la integración de las políticas públicas de mitigación y adaptación al cambio climático, con los objetivos de las políticas sectoriales, como son - por ejemplo - los temas de energía, pobreza, desarrollo económico, desigualdad, pueblos indígenas, salud y desastres.

Adicionalmente, hay una serie de problemas que emergen junto con la integración de políticas y que, en general, tienen que ver con la relación entre la eficiencia y la efectividad de esas políticas de mitigación y adaptación, y su relación con valores éticos fundamentales como la justicia y la igualdad en la aplicación de esas políticas. Esta integración es necesaria para conseguir una relación armoniosa y coherente entre las políticas públicas en general y, particularmente, respecto de políticas públicas específicas, como pueden ser, por ejemplo, las relativas al sistema y cambio de uso de los suelos, la agricultura y la actividad forestal. Las políticas sectoriales deben apropiarse de los objetivos, metas y acciones de las políticas públicas en materia de cambio climático. Obviamente que ésta no es una tarea fácil en vista que los actores involucrados, las escalas temporal y espacial en las que se presentan, y las prioridades que se les asignan, son usualmente distintas en el caso de las medidas de mitigación y adaptación (Di Gregorio et al., 2016, p. 10). En ese sentido, cabe la posibilidad de que ambas puedan tener eventualmente interacciones que produzcan resultados negativos al perjudicar o dificultar la implementación de medidas.

Sobre esto último, hay que recordar que las políticas de mitigación, están orientadas a satisfacer objetivos de largo plazo y contribuir a una reducción de las emisiones de carbono a nivel global. Las políticas de adaptación, en cambio, pueden ser tanto de corto cuanto de largo plazo y pretenden beneficiar a los ecosistemas locales y a las personas que viven en esos lugares (Di Gregorio et al., 2016, p. 10). Esto es sumamente importante cuando se plantea el tema de la gobernanza climática en la Amazonía, porque estamos hablando de dos conjuntos de políticas de intervención del cambio climático que tienen objetivos, espacios y tiempos de aplicación distinta.

Entonces emerge la cuestión de:

1) ¿cómo asegurar la coherencia y la integración de esas políticas que son dependientes de variables globales, y que está más allá de la capacidad de manejo y control de los países amazónicos? y, de otra parte,

2) ¿cómo asegurar la coherencia y la integración de esas políticas cuando éstas son altamente diferenciadas, porque el bioma amazónico es complejo, amplio y diverso, por lo que no son únicamente políticas "nacionales" o "locales"? 
La coherencia e integración de políticas es importante en la construcción de un sistema de gobernanza. Pero conseguir la coherencia y la integración de las políticas no es un proceso fácil o siempre viable en la práctica. Ambas suponen riesgos que, según Thuy et al. (2014) pueden implicar una mayor complejidad que dificulte la formulación de dichas políticas. También existe el peligro de que el proceso de toma de decisiones termine siendo más prolongado y que el deseo de incluir a varios actores distintos, cree expectativas y haga imposible escoger opciones eventualmente excluyentes. Esto último es vital, porque no siempre es posible compatibilizar los objetivos de la mitigación con aquéllos de la adaptación, por la existencia de conflictos de interés y de una escala de valores en juego. La existencia de instituciones sólidas emerge como una cuestión central si se quiere superar esas diferencias y conflictos, y estar en condiciones de avanzar en las medidas de adaptación, incluyendo la asignación de bienes comunes y la adecuación de las leyes y regulaciones (Bisaro, 2016).

Hay otros temas de fondo que afectan la coherencia y las posibilidades de éxito de las políticas integradas de mitigación y adaptación en la Amazonia. Uno de ellos es la ausencia de un ordenamiento territorial en función de los intereses de los pueblos indígenas y comunidades tradicionales locales y que, usualmente, toma sólo el interés del poder central. El otro, es la falta de seguridad jurídica de estos grupos humanos respecto a la propiedad de la tierra. En un contexto en el que estos pueblos encuentran su derecho a la tierra cuestionado o subordinado a los intereses de los grupos de poder transnacional y local, se genera una superposición de posesiones sobre la tierra, se estimula acciones criminales como el narcotráfico y la tala y la minería ilegales, y se genera el desorden y el caos. En esas condiciones es difícil esperar que las políticas de mitigación y adaptación puedan integrarse coherentemente a las políticas públicas sociales y económicas, porque simplemente éstas pueden no existir o ser totalmente débiles e ineficientes.

Según Altomonte et al. (2016, pp. 251-258), que analiza la "buena gobernanza" con relación a la explotación responsable de los recursos naturales en América Latina, es necesario "aplicar buenas prácticas, con sólidas políticas y leyes que prioricen el respeto de las poblaciones asociadas a los proyectos extractivos y la preservación de la naturaleza, en particular de los factores más sensibles relacionados con las actividades productivas de las comunidades locales, particularmente en relación con el agua, el suelo y el entorno natural", siendo que la buena gobernanza debe garantizar el tránsito hacia un paradigma de desarrollo con igualdad.

Finalmente, la coherencia y la integración de las políticas públicas basadas en la mitigación y adaptación no se agotan en los problemas antes mencionados, sino que se relacionan con el tema del financiamiento climático. El tema del financiamiento climático, no se aborda solamente desde la perspectiva de la movilización de los recursos financieros necesarios - que son siempre insuficientes - sino también por la forma en los que estos son puestos a disposición de los países y actores que los requieren. Hay toda una nueva condicionalidad y la existencia de trabas burocráticas y técnicas que obstaculizan el proceso. El empoderamiento de las organizaciones regionales - como es el caso de la Organización del Tratado de Cooperación Amazónica (OTCA) - como entidades movilizadoras del financiamiento y agencias implementadoras, puede contribuir a reducir esos cuellos de botella y hacer del financiamiento climático una herramienta más eficiente y efectiva.

\section{El Acuerdo de París y la Amazonia}

El Acuerdo de París (APCC) es un hito en el proceso colectivo destinado a conseguir la reducción de las emisiones de $\mathrm{CO}_{2}$ y su concentración en la atmósfera, como causantes del calentamiento global. En el APCC se acordó el objetivo de limitar el aumento de la temperatura por debajo de los $2^{\circ} \mathrm{C}$, comparado con los niveles pre- industriales, y tomar acciones para mantener el calentamiento global en $1,5^{\circ} \mathrm{C}$. Mantenerse por debajo de los $2^{\circ} \mathrm{C}$ obligaría a que las concentraciones de GEl se estabilicen en no más de 450 ppm y las emisiones globales alcancen un pico y luego caigan en un 40 o 70\% para el año 2050 (Bodansky, 2017, pp. 5-6). Asimismo, el APCC reconoce la importancia de los bosques como sumideros de carbono y su papel en la conservación de los ecosistemas. 
El APCC, que es un tratado jurídicamente vinculante para todos los países signatarios, entró en vigencia el 4 de noviembre de 2016. Se ha destacado entre sus virtudes el ser lo suficientemente flexible para atender a los diferentes intereses de los países en desarrollo e industrializados. Sin embargo, esto mismo puede ser justamente una debilidad en el largo plazo, en vista de la gravedad del cambio climático. La verdad es que, se trate el APCC de un acuerdo flexible o no, poco o nada puede hacerse sin la voluntad, confianza, reciprocidad y conducta transparente entre los estados.

Asimismo, en el marco del principio de la igualdad entre países emisores y no emisores, con un criterio de flexibilidad se acordó ratificar, como instrumento principal del APCC, la presentación de las Contribuciones Determinadas a nivel Nacional (CDN/NDC en inglés) en las cuales los estados signatarios comunicarán, de manera progresiva cada cinco años, las medidas ambiciosas de mitigación y adaptación que adopten para conseguir detener el calentamiento global hacia mediados del siglo.

Este compromiso se ha establecido "de modo que refleje la equidad y el principio de las responsabilidades comunes pero diferenciadas y las capacidades respectivas, a la luz de las diferentes circunstancias nacionales" (APCC, 2015, artículo 2).

La igualdad que, es una novedad respecto al CMNUCC, establece que todos los países tienen un compromiso en los CDN/NDC, sean grandes o pequeños emisores de $\mathrm{CO}_{2}$. El principio de las responsabilidades comunes pero diferenciadas es un concepto que ya existía y que reconoce que la mayor responsabilidad en el calentamiento global la tienen los países industrializados. Desde comienzos de los años 1990s, los países en desarrollo han reclamado en el sentido de que su obligación de restringir emisiones es menor. Por ello, durante las negociones previas al Protocolo de Kioto y al APCC, se habían opuesto a que se les imponga la obligación de adoptar metas de mitigación (Held, et al., 2013, p. 2). Esta actitud explicaría por qué la posición de los países en desarrollo "ha sido tradicionalmente vista como menos proactiva que aquéllos del mundo industrializado" (Held et al., 2013, p. 2).

Sin embargo, varios países en desarrollo fueron posteriormente flexibilizando su posición negociadora a partir de la Plataforma de Durban para una Acción Reforzada (2011) y formulando de manera voluntaria sus Contribuciones Previstas Determinadas a nivel Nacional (CPDN/INDC), las que fueron interpretadas como una señal positiva.

Asimismo, el APCC incorpora los conceptos de "capacidades respectivas" y "circunstancias nacionales". Ambos conceptos otorgan flexibilidad a los países para la implementación del APCC. Si bien estos conceptos permitieron romper resistencias en la negociación y llegar a un acuerdo, tienen el riesgo de ofrecer una puerta de escape para evadir las obligaciones.

Si bien puede resultar complejo frente a la urgencia de enfrentar el calentamiento global, el APCC preserva el respeto a la soberanía y autonomía de los estados. Lo hace limitando esos compromisos a las capacidades de los países, a la luz de las diferentes circunstancias nacionales. Ese es el sentido en el que el APCC se refiere a las Contribuciones Determinadas a nivel Nacional (CDN/NDC) de los países en desarrollo, (Klein et al, 2017, pp. 3009-3013). Como se ha dicho, además estas contribuciones deberán ser compromisos ambiciosos, aunque progresivos, conforme al artículo 3 del APCC. En el caso de los países menos adelantados o pequeños estados insulares, esas circunstancias son calificadas como especiales.

Es también importante destacar que, junto con los conceptos ya existentes de mitigación y adaptación, el APCC incorporó, en el artículo 8, el reconocimiento de:

la importancia de evitar, reducir al mínimo y afrontar las pérdidas y los daños relacionados con los efectos adversos del cambio climático, incluidos los fenómenos meteorológicos extremos y los fenómenos de evolución lenta, y la contribución del desarrollo sostenible a la reducción del riesgo de pérdidas y daños (APCC, 2015, pp. 8-9). 
Este artículo, a aplicarse en el marco del Mecanismo Internacional de Varsovia bajo la autoridad de la COP, reconoce la importancia de la cooperación para enfrentar las pérdidas y daños que el cambio climático ya está provocando, especialmente en aquellos países que son más vulnerables por su ubicación geográfica y sus niveles de desarrollo.

Un aspecto importante y que marca un cambio de orientación, es que el APCC establece una relación de continuidad y no de oposición entre las medidas de mitigación y de adaptación. En el pasado ambos conceptos fueron manejados en la negociación como opuestos. Por un lado, los países industrializados que debían aumentar sus compromisos de mitigación para atender a su responsabilidad de haber sustentado su desarrollo industrial con el uso de combustibles fósiles, contribuyendo con la emisión de $\mathrm{CO}_{2}$. Por otro lado, los países en desarrollo que no podían asumir compromisos de mitigación, porque no eran responsables del calentamiento global, pero si eran perjudicados por éste.

En el año 2020 los estados signatarios del APCC deberán presentar informes sobre los avances en sus CND/ NDC, incluyendo los compromisos en cuanto a la disminución de las emisiones, las medidas de adaptación ejecutadas y los recursos financieros comprometidos.

Respecto de los anteriores acuerdos del régimen de cambio climático, el APCC fortalece el tratamiento a los conceptos de vulnerabilidad y de resiliencia. El APCC formula como objetivo la adaptación frente a los efectos adversos del cambio climático, mediante el fortalecimiento de las capacidades de adaptación y de resiliencia, así como la reducción de los niveles de vulnerabilidad. Esto es un reconocimiento al hecho de que los efectos adversos del calentamiento global se están sintiendo y son parte de las noticias diarias sobre eventos climáticos extremos. Es una muestra de preocupación sobre el poco avance en la reducción de las emisiones de $\mathrm{CO}_{2}$. Por ejemplo, hace pocos días, el diario británico The Guardian ${ }^{7}$, haciendo eco de las conclusiones del Sexto Informe de Evaluación del Cambio Climático (IPCC) que está siendo lanzado, informaba que las emisiones de $\mathrm{CO}_{2}$ "aumentaron un 1,6\% en 2017 después de tres años en que habían aumentado poco o nada”. Asimismo, contrariamente al compromiso de descarbonización asumido por los países, la "demanda de petróleo está aumentando alrededor de $1.5 \%$ por año".

La naturaleza progresiva de los altos niveles de ambición contenidos en el APCC, sugiere la posibilidad de avanzar en el diseño de un modelo que tome en cuenta simultáneamente el desarrollo, la disminución de las emisiones de $\mathrm{CO}_{2}$, el papel de sumidero de carbono de los ecosistemas, y la necesidad de fortalecer la resiliencia social-ecológica. Especial tratamiento se contempla para los estados insulares y menos desarrollados, para lo cual se ha planteado la meta de movilizar 100 mil millones de dólares anuales desde el año 2020, utilizando para ello los recursos disponibles de varias fuentes de financiamiento. En el caso de la Amazonía, esto implica el reforzamiento de la gestión integrada y sostenible de los recursos forestales, incluyendo el monitoreo de la cobertura forestal, la reducción de la deforestación, del cambio del uso del suelo y de la degradación ambiental, como fenómenos que incrementan la emisión de $\mathrm{CO}_{2}$ y reducen la función del bosque y de los suelos como sumideros de carbono.

EI APCC establece que los países en desarrollo deberán contribuir al financiamiento climático, lo que es una variación con la experiencia previa. Sin embargo, este compromiso está plenamente alineado con la Agenda de Acción de Addis Abeba (AAAA) aprobada en la Tercera Conferencia sobre el Financiamiento para el Desarrollo en diciembre de 2015 (AAAA, 2015). En el AAAA se estableció que el financiamiento para el desarrollo debe provenir de todas las fuentes, públicas y privadas, bilaterales y multilaterales, y fuentes alternativas de financiación.

Asimismo, en el APCC se acordó la introducción de metodologías transparentes para la presentación de informes sobre la financiación para el cambio climático, y el compromiso del Fondo Verde para el Clima (FVC) de destinar un $50 \%$ de sus recursos para proyectos de adaptación, entre otras cuestiones. Igualmente 
se estableció el compromiso de financiar el fortalecimiento de las capacidades de los agentes nacionales y locales para gestionar los riesgos financieros y de desastres.

Es indudable que la plena implementación del APCC requerirá una verdadera revolución económica que mude la matriz energética basada en el uso de combustibles fósiles hacia una que utilice energías renovables. Esa transformación, que no será posible sólo con medidas paliativas, va a requerir nuevas herramientas tecnológicas y financieras que sirvan para ese propósito (Klein et al., 2017, pp. 41-45)

El acuerdo por sí solo no resuelve las grandes cuestiones que están en juego. Su implementación deberá tomar en cuenta las experiencias pasadas que se han tenido con el CMNUCC y el Protocolo de Kioto en los últimos 26 años, para pasar de las formulaciones generales del texto del acuerdo, al establecimiento de reglas y procedimientos específicos necesarios para su implementación, desarrollando visiones compartidas y manteniendo los niveles de ambición alcanzados (Klein et al., 2017, pp. 1784-1788). Este proceso implicará necesariamente avanzar en la superación de las desconfianzas mutuas, mediante la armonización de los intereses individuales de los países signatarios, el uso de mecanismos transparentes que fortalezcan los intereses colectivos, estimulando la reciprocidad y superando el dilema social de la "Tragedia de los Comunes".

Otra característica de la flexibilidad del APCC es que, en su artículo 14, se estableció el compromiso de que la Conferencia de las Partes (COP) efectuaría la evaluación del "balance mundial" del progreso colectivo, y no del individual, hacia los objetivos de largo plazo. El tema fue materia de un arduo debate, en vista que implicaba colocar bajo el escrutinio de la COP, los avances en el cumplimiento de los compromisos, siendo que varios países deseaban preservar su autonomía y la posibilidad de decidir a nivel nacional sus contribuciones. El artículo 14 del APCC refleja un compromiso entre el respeto a la autonomía nacional y el seguimiento robusto de los cumplimientos. Por ello se concordó que serían analizados los avances colectivos y no los individuales, manteniendo así las libertades y flexibilidades necesarias entre países tan diversos, tomando en cuenta aspectos centrales como la equidad y los informes científicos (Klein et al., 2017, pp. 3043-3051).

La fórmula arriba descrita es eminentemente flexible y fue, con seguridad, la única manera de avanzar en las negociaciones hacia un acuerdo, aunque al dejar el tema a la discrecionalidad de los países signatarios, se hace necesario una presencia fortalecida de las instituciones del APCC para asegurar la implementación y el espíritu de confianza, reciprocidad y cooperación. La incorporación de dichos CND/NDC dentro de las políticas y legislaciones nacionales resulta fundamental para asegurar la cooperación internacional.

Entonces, la adaptación a los efectos adversos del cambio climático va a requerir también la adaptación de las instituciones del estado a esa nueva realidad. Se requiere una estructura institucional renovada, policéntrica, coherente y sólida, pero al mismo tiempo flexible, en la que se pueda encontrar visiones compartidas de los problemas y sus posibles soluciones. Asimismo, que puedan implementar mecanismos que faciliten la toma de decisiones colectivas, así como de coordinación y canales para el diálogo.

Esas instituciones deberán tener la flexibilidad para adaptarse a los cambios que puedan presentarse de modo previsible o inesperado, como ocurrió con las sequías severas de los años 2005 y 2010 (Dalagnol et al., 2017, p. 214; Fearnside, 2009, p. 1005) que aislaron y colocaron en una situación de emergencia a las comunidades amazónicas.

En ese proceso, interactúan el conocimiento adquirido y compartido colectivamente, los avances tecnológicos y el intercambio de información, elementos que se convierten en una base común para la formulación de políticas. Los ideales políticos y sociales como democracia, paz, solidaridad, equidad deberían ser parte de esa estructura institucional (Bennet, 2004, p. 49; Armitage et al., 2012, p. 248). 


\section{Reflexiones finales}

La crisis ambiental y climática global han llevado al sistema planetario Tierra a un punto de no retorno en el que las condiciones de vida se ven comprometidas. Dicha crisis afecta gravemente a la Amazonía y pone por delante una seria de desafíos que será necesario enfrentar y que se prevé puedan llevarla a su conversión en una Sabana a mediados de este siglo (Nobre, 2014, p. 27).

Por ello se ha planteado la necesidad de una nueva conceptualización de los problemas descritos, mediante la formulación de la Tierra y de la Amazonía como unidades de análisis y de gestión, entendidas como sistemas social-ecológicos complejos. Esta conceptualización se basa en la relación Tierra-Amazonía y Amazonía-Tierra, y en el concepto de resiliencia cuya construcción ha devenido en urgente frente a las amenazas que se ciernen sobre ellas.

Esta nueva conceptualización debería ayudar en una aproximación holística hacia la construcción de políticas públicas que integren coherentemente a las políticas de mitigación y de adaptación en la Amazonía. El enfoque de paisajes culturales y el reconocimiento del papel transformador de la mujer y el hombre amazónicos y su cultura sobre el mismo, es una cuestión fundamental.

Si no se considera la transformación de las limitaciones estructurales y el desarrollo económico y social como tareas prioritarias, de modo coherente e integrado con las políticas de mitigación y adaptación al cambio climático, poco o nada se podrá avanzar realmente en este último. 


\section{NOTAS}

1 “Evapotranspiración: La evaporación y la transpiración forman los principales flujos de humedad lejos de la superficie de la Tierra. Como rara vez podemos ver los procesos que se llevan a cabo, es fácil descuidar este componente del ciclo hidrológico, pero es extremadamente importante. Devuelve humedad al aire, reponiendo la pérdida por precipitación, y también juega un papel en la transferencia global de energía." (Smithson et al., 2008, pp. 3149-3153).

2 "Cuando deseamos enfatizar la integración concepto de los seres humanos en la naturaleza, utilizamos los términos sistema socio-ecológicos y vínculos socialecológicos, de acuerdo con nuestro trabajo anterior (Berkes y Folke, 1998). Sostenemos la opinión de que los sistemas sociales y ecológicos están de hecho vinculados, y que la delimitación entre los sistemas sociales y naturales es artificial y arbitraria." (Berkes et al., 2003, pp. 200-205)

3 "La palabra tradicional significa cal histórica y continuidad cultural, pero al mismo tiempo reconocemos que las sociedades están en un proceso dinámico de cambio, redefiniendo constantemente lo que se considera "tradicional" (Berkes et al., 2003, pp. 335-339)

4 Véase [https://www.natura-medioambiental.com/cuales-la-importancia-de-la-amazonia/].

5 "En toda la región se ha constatado una espiral de conflictos que ha erosionado la democracia y la cohesión social de los países y comprometido la responsabilidad internacional de los Estados. En un estudio dirigido por Stavenhagen (2006) se identificaron 226 conflictos socioambientales relacionados con proyectos extractivos en territorios indígenas de América Latina en el período 2010-2013 (véase el mapa IV.1)". (Altomonte et al., 2016, p. 225).

6 Traducido de la palabra portuguesa várzeas

7 Véase [https://www.theguardian.com/ commentisfree/2018/sep/30/the-guardian-view-onclimate-policy-failures-dont-give-up]. Consultado [3009-2018].

\section{REFERENCIAS BIBLIOGRÁFICAS}

Altomonte, H. \& Sánchez, R. J. (2016). Hacia una nueva gobernanza de los recursos naturales en América Latina y el Caribe. Santiago de Chile: CEPAL.

Armitage, D., Loe, R., \& Plummer, R. (2012). Environmental governance and its implications for conservation practice. Conservation Letters, 5, 245-255.

Becker, B. K. (2013). Amazônia: mudança climática, projetos globais e interesse nacional. Parcerias Estratégicas, 18(36), 107-128.

Bennett, G. (2004). Nepal: The Terai Arc Landscape Developing an Ecological Network in a Poor, Densely Populated Region. En Bennett, G. Integrating biodiversity conservation and sustainable use: lessons learned from ecological networks. IUCN.

Berkes, F., Colding, J. \& Folk, C. (2003). Navigating Social - Ecological Systems. Building Resilience for Complexity and Change. Cambridge: University Press.

Biermann, F. (2014). Earth system governance: world politics in the anthropocene. Massachusetts, London: The MIT Press Cambridge.

Biermann, F., Pattbergm P. \& Zelli, F. (2010). Global Climate Governance beyond 2012: Architecture, Agency and Adaptation. Cambridge: University Press.

Bisaro, A. \& Hinkel, J. (2016). Governance of social dilemmas in climate change adaptation. Nature Climate Change, 6. Recuperado de [www.nature.com/ natureclimatechange].

Bodansky, D., Brunnée, J. \& Rajamani, L. (2017). International Climate Change Law. Oxford: OUP.

Crutzen, P. J. (2002). The Anthropocene. Geology of Mankind. Nature, 416. Recuperado de [www.nature. com].

Dalagnol, R., Borma, L. S., Mateus, P. \& Rodríguez, D. A. (2017). Assessment of climate change impacts on water resources of the Purus Basin in the southwestern Amazon. Acta Amazónica, 47(3), 213- 226. Doi: http:// dx.doi.org/10.1590/1809-4392201601993.

Deleuze, G. (1994). What Is Philosophy? European Perspectives: A Series in Social Thought and Cultural Criticism. Columbia: University Press.

Di Gregorio, M., Fatorelli, L., Pramova, E., May, P., Locatelli, B. \& Brockhaus, M. (2016). Integrating mitigation and adaptation in climate and land use policies in Brazil: a policy document analysis. Centre for Climate Change Economics and Policy Working Paper $n^{\circ}$ 257. Sustainability Research Institute. CIFOR Working Paper $n^{\circ}$ 194. Economic and Social Research Council, University of Leeds, The London School of Economics and Political Science (LSE). 
Fearnside, P. M. (2009). Global warming in Amazonia: Impacts and Mitigation. Acta Amazónica, 39(4), 10031012.

Gonçalves, C. W. P. (2012). Amazônia, Amazônias. São Paulo: Contexto.

Held, D., Roger, C. \& Nag, E. M. (Eds). (2013). Climate Governance in the Developing World. New Jersey: Wiley.

IPCC. (2014). Cambio climático 2014: Informe de síntesis. Contribución de los Grupos de trabajo I, II y III al Quinto Informe de Evaluación del Grupo Intergubernamental de Expertos sobre el Cambio Climático. Ginebra: IPCC. Recuperado de [https://www.ipcc.ch/site/ assets/uploads/2018/02/SYR_AR5_FINAL_full_es.pdf]. Consultado [14-01-2019].

Jang, Ji., McSparren, J. \& Rashchupkina, Y. (2016). Global governance: present and future. Palgrave Communications, 2, Nature. Doi: https://doi.org/10.1057/ palcomms.2015.45

Klein, D., Carazo, M. P., Doelle, M., Bulmer, J. \& Higham, A. (Eds). (2017). The Paris Agreement on Climate Change: Analysis and Commentary. Oxford: OUP.

Kotzé, Louis J. et al. (2017). Environmental Law and Governance for the Anthropocene. Oxford: Hart Publishing.

Nobre, A. D. (2014). The Future Climate of Amazonia, Scientific Assessment Report. São José dos Campos, AARA. Recuperado de [file:///C:/Users/Elisa/Downloads/ The_Future_Climate_of_Amazonia_Report.pdf]. Consultado [14-01-2019].

Organización de las Naciones Unidas (ONU). (2015). 69/313. Agenda de Acción de Addis Abeba de la Tercera Conferencia Internacional sobre la Financiación para el Desarrollo (Agenda de Acción de Addis Abeba). Asamblea General. Sexagésimo noveno período de sesiones. Recuperado de [http://unctad.org/meetings/es/ SessionalDocuments/ares69d313_es.pdf]. Consultado [22-09-2018].

Organización de las Naciones Unidas (ONU). (2015). Acuerdo de París. Recuperado de [https://unfccc.int/ files/meetings/paris_nov_2015/application/pdf/paris agreement_spanish_.pdf]. Consultado [147-01-2019].

PNUMA \& OTCA. (2009). Geoamazonía. Perspectivas del Medio Ambiente en la Amazonía. Ciudad de Panamá: PNUMA y OTCA. Recuperado de [http://www.pnuma. org/deat1/pdf/geoamazonia_spanish_FINAL.pdf].

Rockström, J., et. al. (2009). Planetary boundaries: exploring the safe operating space for humanity. Ecology and Society 14(2), 32. Recuperado de [http://www. ecologyandsociety.org/vol14/iss2/art32/].

Smithson, P., Addison, K. \& Atkinson, K. (2008). Fundamentals of the Physical Environment. London: Routledge.

Tanner, T. \& Phathanothai, L. H. (2014). Climate Change and Development. London: Routledge.
Thuy, P. T, et. al. (2014). Integration of Adaptation and Mitigation in Climate Change and Forest Policies in Indonesia and Vietnam. Forests, 5(8), 20162036. Recuperado de [http://www.mdpi.com/19994907/5/8/2016/htm]. Consultado [18-09-2018].

UICN. (2008). Indigenous and Traditional peoples and Climate Change. Issues Paper.

UNEP/ACTO. (2009) GEO AMAZONIA: Environment Outlook in the Amazonia. United Nations Environment Programme (UNEP), the Amazon Cooperation Treaty Organization (ACTO) and Research Centre of the Universidad del Pacifico (CIUP). Recuperado de [http://wedocs.unep.org/handle/20.500.11822/9421]. Consultado [29-07-2017].

World Bank. (2010). World Development Report 2010 - Development and Climate Change. The International Bank for Reconstruction and Development. Recuperado de [http://www.worldbank.org/wdr]. Consultado [28-072017].

Young, O. R. (2017). Governing Complex Systems: Social Capital for the Anthropocene (Earth System Governance). Cambridge: MIT Press. 